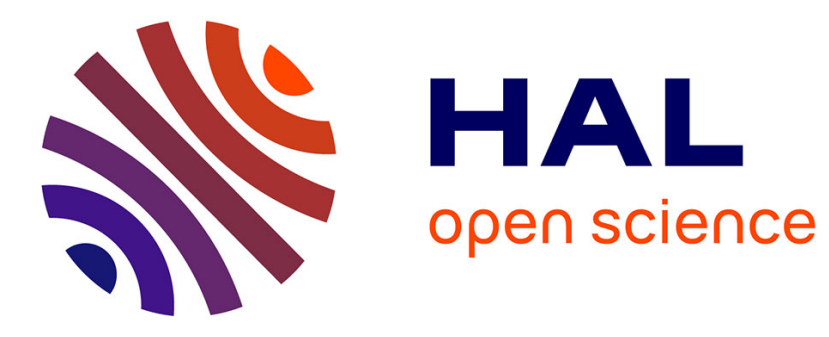

\title{
Analysis of heavily boron-doped diamond Raman spectrum
}

\author{
V. Mortet, A. Taylor, Z. Vlčková Živcová, D. Machon, O. Frank, P. Hubík, \\ David Trémouilles, L. Kavan
}

\section{- To cite this version:}

V. Mortet, A. Taylor, Z. Vlčková Živcová, D. Machon, O. Frank, et al.. Analysis of heavily boron-doped diamond Raman spectrum. Diamond and Related Materials, 2018, 88, pp.163-166. 10.1016/j.diamond.2018.07.013 . hal-01956849

\section{HAL Id: hal-01956849 \\ https://hal.laas.fr/hal-01956849}

Submitted on 11 Feb 2019

HAL is a multi-disciplinary open access archive for the deposit and dissemination of scientific research documents, whether they are published or not. The documents may come from teaching and research institutions in France or abroad, or from public or private research centers.
L'archive ouverte pluridisciplinaire HAL, est destinée au dépôt et à la diffusion de documents scientifiques de niveau recherche, publiés ou non, émanant des établissements d'enseignement et de recherche français ou étrangers, des laboratoires publics ou privés. 


\title{
Analysis of heavily boron-doped diamond Raman spectrum
}

\author{
V. Mortet ${ }^{\mathrm{a}, \mathrm{b}, *}$, A. Taylor ${ }^{\mathrm{a}}$, Z. Vlčková Živcová ${ }^{\mathrm{c}}, \mathrm{D}$. Machon ${ }^{\mathrm{d}}$, O. Frank ${ }^{\mathrm{c}}$, P. Hubík ${ }^{\mathrm{e}}$, D. Tremouilles ${ }^{\mathrm{f}}$, \\ L. Kavan ${ }^{\mathrm{c}}$ \\ a Institute of Physics of the Czech Academy of Sciences, Na Slovance 1999/2, 18221 Prague 8, Czech Republic \\ ${ }^{\mathrm{b}}$ Czech Technical University in Prague, Faculty of Biomedical Engineering, Sítna 3105, 27201 Kladno, Czech Republic

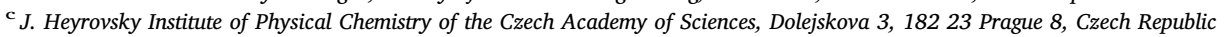 \\ ${ }^{\mathrm{d}}$ Institut Lumière Matière, Université de Lyon, Université Claude Bernard Lyon 1, CNRS UMR 5306, 69622 Villeurbanne, France \\ ${ }^{\mathrm{e}}$ Institute of Physics of the Czech Academy of Sciences, Cukrovarnicka 10, 16200 Prague 6, Czech Republic \\ ${ }^{\mathrm{f}}$ LAAS-CNRS, Université de Toulouse, 7, avenue du Colonel Roche, 31031 Toulouse Cedex 4, France
}

\section{A B S T R A C T}

Lattice disorder, electronic Raman scattering, and Fano interaction effects are at the genesis of the Raman spectrum of heavily boron-doped diamond. However, no accurate unified description of this spectrum has been reported yet. In this work, we propose a novel analysis of the Raman spectrum of boron-doped diamond based on classical models of electronic Raman scattering and Fano effect. This new analysis shows that the Raman spectrum of boron-doped diamond results from the combination of electronic Raman scattering and its interaction, i.e. Fano effect, with the diamond phonon density of states and it confirms the $500 \mathrm{~cm}^{-1}$ and $1200 \mathrm{~cm}^{-1}$ bands originate from the phonon density of states.

\section{Introduction}

Raman spectroscopy is a fast and nondestructive technique widely used for the characterization of diamonds. Intensities, position and full width at half maximum of Raman peaks are representative of the quality and composition of diamond layers. For instance, heavily borondoped diamond with metallic electrical conductivity has a characteristic Raman signature with two broad bands at ca. $500 \mathrm{~cm}^{-1}$ (B1) and ca. $1200 \mathrm{~cm}^{-1}$ (B2) along with a Fano-shaped zone-center phonon (ZCP) peak below $1332 \mathrm{~cm}^{-1}$. It is conjectured the Fano-shape results from interaction between discrete diamond zone-center phonon (ZCP) and electronic Raman scattering effects. The majority of studies on the Fano-shaped diamond peak reports a negative value for the Fano asymmetric parameter (q) [1-7]. Other authors reported positive values of the Fano asymmetric parameter in layers [2, 8, 9] with boron concentrations close to the metal-insulator transition (Mott transition). The origin of other Raman peaks is subject to discussion. The B2 band had previously been attributed to vibration modes of boron atoms [10]. However, substitutional isotopic studies have, unambiguously, demonstrated its vibration origin to be related to carbon atoms [1, 11, 12]. It is generally agreed that this band corresponds to a maximum of the diamond phonon density of states (PDoS) $[13,14]$. The appearance of this Raman forbidden band is due to the relaxation of the Raman wave vector conservation rule in materials with high lattice disorder [7, 15], i.e., the high concentration of substitutional boron atoms in the diamond lattice. However, this interpretation is not fully satisfactory due to the mismatch between the experimental peak position, the known optical maxima of diamond's PDoS [16] and contradictory reports of the peak shape: Szirmai et al. presented this peak as symmetrical with a Lorentzian shape [1], whereas Bustarret et al. depicted it as an asymmetric Fano-shaped peak [3, 17]. The broad asymmetric band B1 is empirically modeled with the sum of a Lorentzian and a Gaussian [18] or a Lorentzian component [1]. It is very sensitive to the boron concentration [19, 20] and it was attributed to vibration modes of boron dimers [18, 21]. However, substitutional isotopic studies have failed to unambiguously confirm this assignment $[12,14]$. The purpose of this article is to address these contradictions. Based on classic models of electronic Raman scattering, Fano effect, and analytical methodology we clarify the origin of the Raman spectrum of the boron-doped diamond.

\section{Experimental}

In order to remove any added complication due to the presence of $\mathrm{sp}^{2}$ carbon phase Raman features, which are present in polycrystalline diamond layers, this study has been carried out on epitaxial borondoped diamond layers. The studied epitaxial layers were grown on high pressure and high-temperature diamond substrates in a $1.5 \mathrm{~kW}$ resonance cavity microwave plasma enhanced chemical vapor deposition system, AX5010 from Seki Diamond Systems, with two different boron

\footnotetext{
* Corresponding author at: Institute of Physics of the Czech Academy of Sciences, Na Slovance 1999/2, 182 21 Prague 8, Czech Republic.

E-mail address: mortetv@fzu.cz (V. Mortet).
} 
concentrations. Sample S1 was grown with a boron to carbon ratio, in the gas phase, of $4000 \mathrm{ppm}$ on a (100) oriented substrate using deposition conditions reported in ref. [22], whereas sample S2 was grown with a higher boron to carbon ratio of $40,000 \mathrm{ppm}$ on a (111) oriented substrate using deposition conditions reported in ref. [20]. Raman spectra were measured using a LabrRAM HR spectrometer from Horiba Jobin-Yvon interfaced to an Olympus microscope with a $100 \times$ objective and a $50 \mu \mathrm{m}$ confocal aperture, and a HeNe laser $633 \mathrm{~nm}(1.96 \mathrm{eV})$ with $8 \mathrm{~mW}$ power. The spectrometer was calibrated using the silicon $\mathrm{F}_{1 \mathrm{~g}}$ peak at $520.2 \mathrm{~cm}^{-1}$ and its spectral response was calibrated using a certified HL-2000 Tungsten Halogen Light Source from Ocean Optics. Raw Raman spectra were corrected for instrumental response, temperature effect and the frequency factor [23-25]. The temperature correction is particularly important in order to observe the broad electronic Raman scattering response. Corrected spectra or reduced Raman $\left(I_{r}(\omega)\right)$ spectra are calculated using Eq. (1) with $I(\omega)$ the experimental spectrum, $K(\omega)$ the spectrometer response; $\omega_{L}$ the absolute frequency of laser excitation line, $\omega$ the Raman shift, and $n(\omega)+1$ the thermal population factor with $n(\omega, \mathrm{T})$ the Bose-Einstein distribution.

$I_{r}(\omega)=I(\omega) \cdot\left[K(\omega) \cdot\left(\omega_{L}-\omega\right)^{4} \cdot \omega^{-1} \cdot(n(\omega, T)+1)\right]^{-1}$

\section{Results and discussion}

Normalized raw and reduced Raman spectrum of both samples are shown in Fig. 1 and Fig. 2, respectively. The raw spectra are characteristic of boron-doped diamond with metallic electrical conductivity with low (sample S1) and high boron concentration (sample S2) [17, 19]. In contrary to sample $S 2$, sample $S 1$ exhibits no measurable band at ca. $500 \mathrm{~cm}^{-1}$. Both reduced spectra retain the characteristic band of boron-doped diamond. They share a broad asymmetric background with a maximum between 1000 and $2000 \mathrm{~cm}^{-1}$ attributed to electronic Raman scattering, a broadband B2 merged with the narrow diamond line, and a broad dip at $1350 \mathrm{~cm}^{-1}$ attributed to Fano antiresonance. The large width of this dip cannot originate only from the antiresonance of the Fano shaped narrow diamond line. We attribute this dip to a combination of the Fano antiresonance of the $\mathrm{B} 2$ band and the diamond line. This assumption is supported by the clear asymmetry of the B2 band, which is characteristic for Fano effect, and is consistent with the work of Bustarret et al. [3]. The two Raman spectra exhibit a different curvature at low wavenumbers, i.e., $<500 \mathrm{~cm}^{-1}$. The concavity of $\mathrm{S} 1$ spectrum is consistent with electronic Raman scattering whereas the convexity of S2 sample may be attributed to a Fano antiresonance of the $\mathrm{B} 1 \mathrm{band}$. This assumption is supported by the well-known asymmetry of

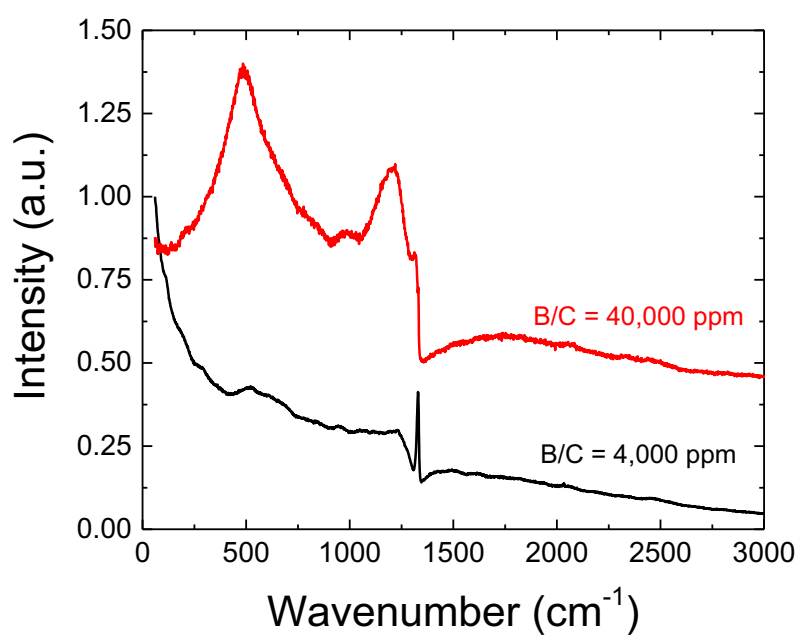

Fig. 1. Normalized raw Raman spectra of boron-doped diamond samples grown at B/C ratios of $4000 \mathrm{ppm}$ (S1) and 40,000 ppm (S2). The spectra of sample S2 is shifted by +0.4 units for improved readability.
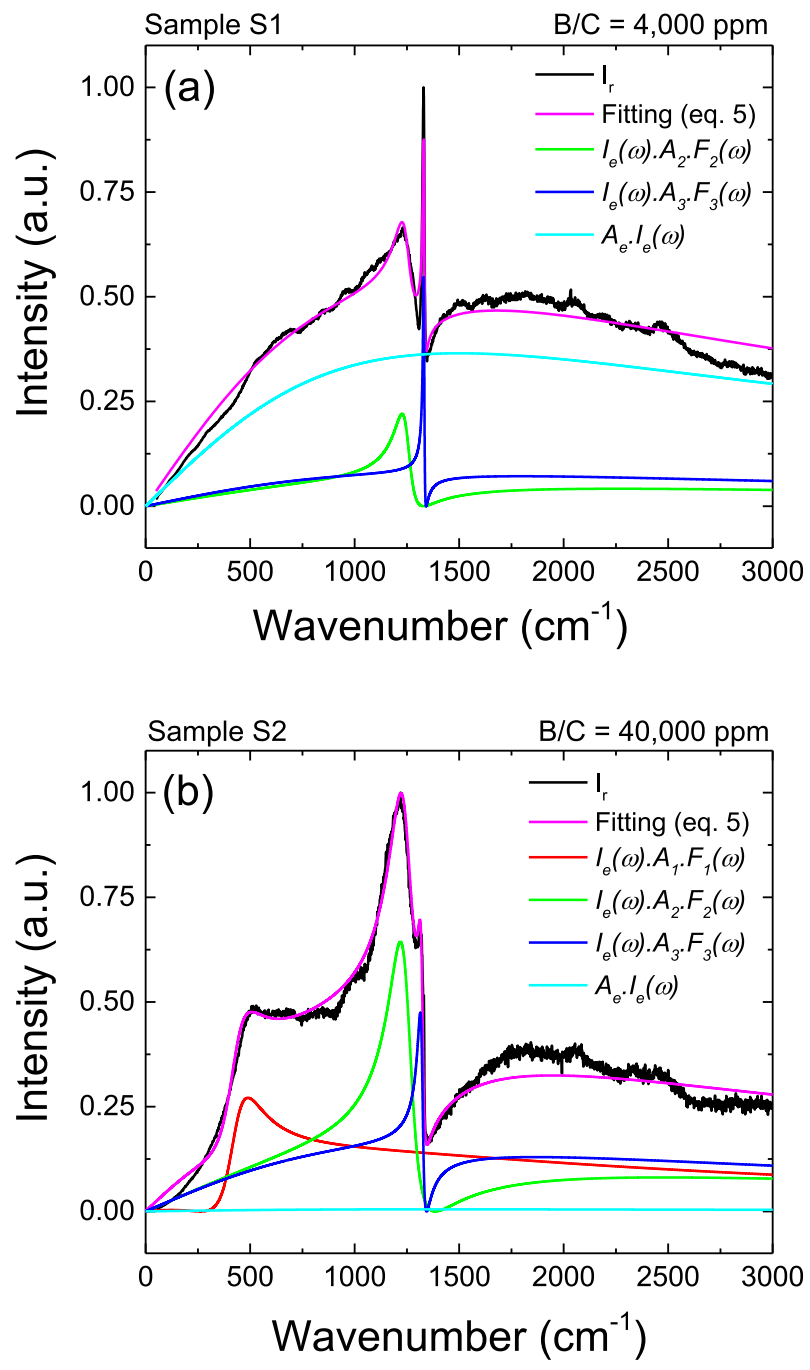

Fig. 2. Experimental reduced and normalized Raman spectra (solid black line) of boron-doped diamond samples grown at B/C ratios of $4000 \mathrm{ppm}$ (S1) and $40,000 \mathrm{ppm}$ (S2), mathematical fittings (magenta solid line) with the contribution of the different Fano shape peaks (red, green and blue lines) and pure electronic Raman scattering broadband (cyan solid line). (For interpretation of the references to colour in this figure legend, the reader is referred to the web version of this article.)

this band. Based on these observations, we constructed a fitting function to describe the Raman spectrum of boron-doped diamond assuming the interaction of the observed Raman lines with electronic Raman scattering applies to the diamond ZCP phonon line and all other observed bands using well-established model of electronic Raman scattering and Fano effect. This hypothesis is legitimized by the asymmetric nature of the different bands and the broad range of electronic Raman scattering.

To our knowledge and in contrary to metals [26, 27] and heavily doped silicon [28], electronic Raman scattering has not been investigated in boron-doped diamond. In this work, the normalized electronic Raman scattering $I_{e}(\omega)$ is modeled using the Eq. (2) used for Raman scattering by intra-band excitations and where $\omega_{e}$ is the position of the maximum of the electronic Raman scattering spectrum. This equation was shown to satisfactorily describe electronic Raman scattering in conventional elemental metals [27] and highly doped semiconductors [29].

$I_{e}(\omega)=\frac{2 \cdot\left(\omega / \omega_{e}\right)}{1+\left(\omega / \omega_{e}\right)^{2}}$ 
Table 1

Fitting parameter values of boron-doped diamond layer Raman spectra. Indices 1,2 , and 3 correspond to the B1 band, B2 band, and ZCP line, respectively.

\begin{tabular}{lll}
\hline Parameter & Sample S1 $(\mathrm{B} / \mathrm{C}=4000 \mathrm{ppm})$ & Sample S2 $(\mathrm{B} / \mathrm{C}=40,000 \mathrm{ppm})$ \\
\hline$\omega_{e}$ & 1400 & 1400 \\
$A_{e}$ & 0.365 & 0.005 \\
$A_{1}$ & - & 0.88 \\
$q_{1}$ & - & 1.83 \\
$\omega_{1}$ & - & 427.2 \\
$\Gamma_{1}$ & - & 90 \\
$A_{2}$ & 0.45 & 1.3 \\
$q_{2}$ & -1.75 & -2.1 \\
$\omega_{2}$ & 1250.9 & 1249.5 \\
$\Gamma_{2}$ & 40.7 & 65 \\
$A_{3}$ & 1.1 & 0.95 \\
$q_{3}$ & -2.5 & -1.5 \\
$\omega_{3}$ & 1332 & 1323.3 \\
$\Gamma_{3}$ & 4.3 & 13.9 \\
\hline
\end{tabular}

Fano shaped peaks are modeled using the normalized Fano function $F_{j}(\omega)$ (see Eqs. (3) and (4)) with $q_{j}$ the Fano asymmetric parameter, $\omega_{j}$ the center position and $\Gamma_{j}$ the full width at half maximum of the Lorentzian shaped Raman lines.

$F_{j}(\omega)=\frac{1}{1+q_{j}^{2}} \frac{\left(q_{j}+\varepsilon_{j}\right)^{2}}{1+\varepsilon_{j}^{2}}$

with

$\varepsilon_{j}(\omega)=\frac{\omega-\omega_{j}}{\Gamma_{j}}$

Based on the assumptions above, the reduced Raman spectrum of boron-doped diamond is fitted using Eq. (5) representing the sum of the different weighted light scattering events: pure electronic Raman scattering and its interaction with the different localized Raman scatterings. $A_{e}$ is the weighted factor of pure electronic Raman scattering and $A_{j}$ are the weighting factor of the different Fano-shaped peaks.

$I_{r}(\omega)=I_{e}(\omega) .\left[A_{e}+\sum_{j} A_{j} . F_{j}(\omega)\right]$

The Raman spectra of diamond samples were fitted with Eq. (5) using the non-linear fitting tool of Origin 2016 (C) software. The fitting function and its different components are reported in Fig. 2, and the fitting parameter is reported in Table 1 . The fitting function satisfactorily models the B2 band, the diamond lines, and antiresonance dip as well as the broad B1 band. It particularly shows that the broad antiresonance dip at ca. $1350 \mathrm{~cm}^{-1}$ is a combination of the diamond ZCP and the B2 bands antiresonances. The Fano asymmetric parameter values of the B2 band are remarkably close to the ones of the ZCP line, which are consistent with values reported in the literature [1-6] and the position and the width of the diamond line are also consistent with our previous report [20]. The fitting peak position of the B2 band $\left(1250 \mathrm{~cm}^{-1}\right)$ corresponds to the reported maxima of diamond PDoS [16] which supports our analysis and confirms the PDoS origin of this band. Similarly, the fitting peak position $\left(427 \mathrm{~cm}^{-1}\right)$ and width $\left(90 \mathrm{~cm}^{-1}\right)$ of the B1 band are comparable with the reported values of the acoustic maximum of PDoS peak measured by Raman spectroscopy on not electrically conductive diamond nanoparticles and disordered diamond $[30,31]$. The assignment of the $\mathrm{B} 1$ band to a maximum of the acoustic branch of the PDoS is consistent with was already hypothesized by several other authors [7, 32]. This analysis indicates that the difference between the apparent position of the B1 band and the position of the maximum of PDoS in disordered diamond is not only due to phonon confinement but it is also due its interaction with electronic Raman scattering, i.e. Fano effect, conformably with the ZCP line [20]. This analysis is also analogous to Yogi et al. work on silicon nanostructures who report the observation of a size-dependent position asymmetric peak due to acoustic phonons by Raman spectroscopy due to the quantum confinement effect and their interaction with intraband quasi-continuum [33].

\section{Conclusion}

In summary, we have shown the Raman spectrum of highly borondoped diamond can be satisfactorily fitted using the combination of well-established electronic Raman scattering and Fano effect models. This analysis shows that not only the diamond ZCP band is Fano-shaped but also the $500 \mathrm{~cm}^{-1}$ and $1200 \mathrm{~cm}^{-1}$ bands. As a result, their apparent position is shifted from their normal position, which corresponds to the acoustic and optical maxima of the phonon density of states in disordered diamond.

\section{Acknowledgment}

This work was financially supported by project 13-31783S of Czech Science Foundation, the French-Czech Project Barrande 35785SC 7AMB16FR004 of the Czech Ministry of Education, Youth and Sports and the J.E. Purkyně fellowship awarded to $V$. Mortet by the Czech Academy of Sciences.

\section{References}

[1] P. Szirmai, T. Pichler, O.A. Williams, S. Mandal, C. Bäuerle, F. Simon, A detailed analysis of the Raman spectra in superconducting boron doped nanocrystalline diamond, Phys. Status Solidi B 249 (2012) 2656-2659, https://doi.org/10.1002/ pssb. 201200461.

[2] K. Ushizawa, K. Watanabe, T. Ando, I. Sakaguchi, M. Nishitani-Gamo, Y. Sato, H. Kanda, Boron concentration dependence of Raman spectra on $\{100\}$ and $\{111\}$ facets of B-doped CVD diamond, Diam. Relat. Mater. 7 (1998) 1719-1722.

[3] E. Bustarret, E. Gheeraert, K. Watanabe, Optical and electronic properties of heavily boron-doped homo-epitaxial diamond, Phys. Status Solidi A 199 (2003) 9-18, https://doi.org/10.1002/pssa.200303819.

[4] F. Pruvost, A. Deneuville, Analysis of the Fano in diamond, Diam. Relat. Mater. 10 (2001) 531-535, https://doi.org/10.1016/S0925-9635(00)00378-2.

[5] C. Piccirillo, A. Mainwood, G. Davies, C.M. Penchina, A. Tajani, M. Bernard, A. Deneuville, The temperature dependence of the infrared absorption and Raman spectra due to boron in diamond, Phys. Status Solidi A 193 (2002) 529-534, https://doi.org/10.1002/1521-396X(200210)193:3<529::AID-PSSA529>3.0. CO;2-5.

[6] P. Gonon, E. Gheeraert, A. Deneuville, F. Fontaine, L. Abello, G. Lucazeau, Characterization of heavily B-doped polycrystalline diamond films using Raman spectroscopy and electron spin resonance, J. Appl. Phys. 78 (1995) 7059-7062, https://doi.org/10.1063/1.360410.

[7] E. Gheeraert, P. Gonon, A. Deneuville, L. Abello, G. Lucazeau, Effect of boron incorporation on the "quality" of MPCVD diamond films, Diam. Relat. Mater. 2 (1993) 742-745, https://doi.org/10.1016/0925-9635(93)90215-N.

[8] R. Locher, J. Wagner, F. Fuchs, M. Maier, P. Gonon, P. Koidl, Optical and electrical characterization of boron-doped diamond films, Diam. Relat. Mater. 4 (1995) 678-683.

[9] A.N. Utyuzh, Y.A. Timofeev, A.V. Rakhmanina, Effect of boron impurity on the Raman spectrum of synthetic diamond, Inorg. Mater. 40 (2004) 926-931.

[10] M. Werner, O. Dorsch, H.U. Baerwind, E. Obermeier, L. Haase, W. Seifert, A. Ringhandt, C. Johnston, S. Romani, H. Bishop, P.R. Chalker, Charge transport in heavily B-doped polycrystalline diamond films, Appl. Phys. Lett. 64 (1994) 595-597, https://doi.org/10.1063/1.111088.

[11] N. Dubrovinskaia, L. Dubrovinsky, T. Papageorgiou, A. Bosak, M. Krisch, H.F. Braun, J. Wosnitza, Large carbon-isotope shift of TC in boron-doped diamond, Appl. Phys. Lett. 92 (2008) 132506, , https://doi.org/10.1063/1.2906381.

[12] P. Achatz, F. Omnès, L. Ortéga, C. Marcenat, J. Vacík, V. Hnatowicz, U. Köster, F. Jomard, E. Bustarret, Isotopic substitution of boron and carbon in superconducting diamond epilayers grown by MPCVD, Diam. Relat. Mater. 19 (2010) 814-817, https://doi.org/10.1016/j.diamond.2010.01.052.

[13] V.A. Sidorov, E.A. Ekimov, Superconductivity in diamond, Diam. Relat. Mater. 19 (2010) 351-357, https://doi.org/10.1016/j.diamond.2009.12.002.

[14] I.I. Vlasov, E.A. Ekimov, A.A. Basov, E. Goovaerts, A.V. Zoteev, On the origin of the Raman scattering in heavily boron-doped diamond, ArXiv Preprint ArXiv 0801.1611 (2008), https://arxiv.org/abs/0801.1611.

[15] K.K. Tiong, P.M. Amirtharaj, F.H. Pollak, D.E. Aspnes, Effects of as + ion implantation on the Raman spectra of GaAs: "spatial correlation" interpretation, Appl. Phys. Lett. 44 (1984) 122-124, https://doi.org/10.1063/1.94541.

[16] A.M. Zaitsev, Optical Properties of Diamond: a Data Handbook, Springer Science \& Business Media, 2013.

[17] S. Ghodbane, A. Deneuville, Specific features of $325 \mathrm{~nm}$ Raman excitation of heavily boron doped polycrystalline diamond films, Diam. Relat. Mater. 15 (2006) 
589-592, https://doi.org/10.1016/j.diamond.2005.12.054.

[18] M. Bernard, C. Baron, A. Deneuville, About the origin of the low wave number structures of the Raman spectra of heavily boron doped diamond films, Diam. Relat. Mater. 13 (2004) 896-899, https://doi.org/10.1016/j.diamond.2003.11.082.

[19] M. Bernard, A. Deneuville, P. Muret, Non-destructive determination of the boron concentration of heavily doped metallic diamond thin films from Raman spectroscopy, Diam. Relat. Mater. 13 (2004) 282-286, https://doi.org/10.1016/j. diamond.2003.10.051.

[20] V. Mortet, Z. Vlčková Živcová, A. Taylor, O. Frank, P. Hubík, D. Trémouilles, F. Jomard, J. Barjon, L. Kavan, Insight into boron-doped diamond Raman spectra characteristic features, Carbon 115 (2017) 279-284, https://doi.org/10.1016/j. carbon.2017.01.022.

[21] E. Bourgeois, E. Bustarret, P. Achatz, F. Omnès, X. Blase, Impurity dimers in superconducting B-doped diamond: experiment and first-principles calculations, Phys. Rev. B 74 (2006), https://doi.org/10.1103/PhysRevB.74.094509.

[22] V. Mortet, L. Fekete, P. Ashcheulov, A. Taylor, P. Hubík, D. Tremouilles, E. BedelPereira, (100) Substrate processing optimization for fabrication of smooth boron doped epitaxial diamond layer by PE CVD, Proceedings of NANOCON 2014, 6th International Conference, 2014, Brno, Czech Republic, EU, November 5-7th, 2014, 2015, pp. 115-119.

[23] R. Carles, M. Bayle, P. Benzo, G. Benassayag, C. Bonafos, G. Cacciato, V. Privitera, Plasmon-resonant Raman spectroscopy in metallic nanoparticles: surface-enhanced scattering by electronic excitations, Phys. Rev. B 92 (2015), https://doi.org/10. 1103/PhysRevB.92.174302.

[24] M.H. Brooker, O.F. Nielsen, E. Praestgaard, Assessment of correction procedures for reduction of Raman spectra, J. Raman Spectrosc. 19 (1988) 71-78.

[25] R. Shuker, R.W. Gammon, Raman-scattering selection-rule breaking and the density of states in amorphous materials, Phys. Rev. Lett. 25 (1970) 222-225, https://doi. org/10.1103/PhysRevLett.25.222.

[26] W. Akemann, A. Otto, Electronic Raman scattering at disordered noble- and alkalimetal surfaces, Philos. Mag. B 70 (1994) 747-760, https://doi.org/10.1080/ 01418639408240247.

[27] Y.S. Ponosov, S.V. Streltsov, Measurements of Raman scattering by electrons in metals: the effects of electron-phonon coupling, Phys. Rev. B 86 (2012), https://doi. org/10.1103/PhysRevB.86.045138.

[28] I.P. Ipatova, A.V. Subashiev, V.A. Voitenko, Electron light scattering from doped silicon, Solid State Commun. 37 (1981) 893.

[29] G. Contreras, A.K. Sood, M. Cardona, Raman scattering by intervalley carrier-density fluctuations in n-type Si: Intervalley and intravalley mechanisms, Phys. Rev. B 32 (1985) 924.

[30] S. Prawer, K.W. Nugent, D.N. Jamieson, J.O. Orwa, L.A. Bursill, J.L. Peng, The Raman spectrum of nanocrystalline diamond, Chem. Phys. Lett. 332 (2000) 93-97.

[31] J.O. Orwa, K.W. Nugent, D.N. Jamieson, S. Prawer, Raman investigation of damage caused by deep ion implantation in diamond, Phys. Rev. B 62 (2000) 5461.

[32] S. Prawer, R.J. Nemanich, Raman spectroscopy of diamond and doped diamond, Philos. Trans. R. Soc. A Math. Phys. Eng. Sci. 362 (2004) 2537-2565, https://doi. org/10.1098/rsta.2004.1451.

[33] P. Yogi, S. Mishra, S.K. Saxena, V. Kumar, R. Kumar, Fano scattering: manifestation of acoustic phonons at the nanoscale, J. Phys. Chem. Lett. 7 (2016) 5291-5296, https://doi.org/10.1021/acs.jpclett.6b02090. 